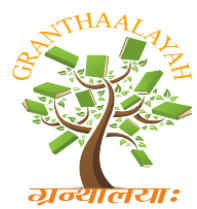

INTERNATIONAL JOURNAL OF RESEARCH GRANTHAALAYAH

A knowledge Repository

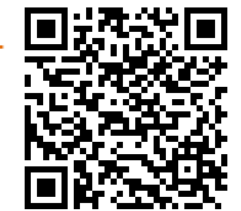

Management

\title{
A STUDY OF INVESTMENT PATTERN ON THE BASIS OF DEMOGRAPHIC TRAITS
}

\author{
Dr. C. M. Shinde *1, Priyanka Zanvar ${ }^{2}$ \\ ${ }^{*}$ Associate Professor, D.G. College of Commerce, Satara, INDIA \\ ${ }^{2}$ Research Scholar, Karmaveer Bhaurao Patil Institute of Management Studies and Research, \\ Satara, INDIA
}

\begin{abstract}
Investor's behavior is influenced by many factors during investment decision making. Demographic profile of investors is also one of the decision influencing factor among others. The aim of this paper is to examine the effect of demographic factors on investor's level of risk tolerance regarding the choice of investment. 670 investors Pune City, Maharashtra State, India were selected as sample. ANOVA, Mann Whiteny ' $U$ ' test, Kruskal- Wallis test were used to explore the effect of demographic factors on investor's level of risk tolerance regarding the choice of investment. Result of the paper showed that demographic factors of investors such as Age, Educational qualification, Income level, effect the investor's level of risk tolerance. These results are important for managers to advise their clients about better area of investment and risk level according to their demographic profile.
\end{abstract}

Keywords:

Demographic traits, Investment Pattern, Investor Behavior.

Cite This Article: Dr. C. M. Shinde, and Priyanka Zanvar, "A STUDY OF INVESTMENT PATTERN ON THE BASIS OF DEMOGRAPHIC TRAITS" International Journal of Research - Granthaalayah, Vol. 3, No. 11(2015): 175-205. DOI: 10.29121/granthaalayah.v3.i11.2015.2927

\section{INTRODUCTION}

Investment has different meaning in the context of finance and economics. Finance investment is putting money into something with the expectation of gain that upon thorough analysis has a high degree of security for the principle amount, as well as security of return, within an expected period of time. In contrast, putting money into something with an expectation of gain without making thorough analysis is speculation or gambling. Thus, Finance Investment involves decision making process in order to ensure security of both the principle amount and the return on investment (ROI) within an expected period of time.

The two main classes of investments are i) Fixed Income Investment such as bonds, fixed deposits, preference shares and ii) Variable Income Investment such as business ownership (equities) or 
property ownership. On the basis of tenure, the investments are classified as i. Short-term Investment and ii. Long-Term Investment. Investments made for a period of one to three years are termed as short-term investments and that are invested for more than three years are termed as long-term investments. Almost everyone holding some portfolio of investment in the form of financial assets like bank deposits, bonds, stocks and so on; and real assets like motorcycle, house, gold etc.

With reference to individuals, investment decisions should be made very wisely and with proper research and analysis. Investment is always attached with the element of risk of losing the invested money and this loss is not under the control of the investor. Hence, it is always advisable to measure and analyze all risks involved before making investments. Plenty of investment avenues available for the investors make their decision making process more critical and complex. There are a number of factors which influence the people to make their investment decisions. Demographic factors of investors such as gender, age, education, family size, annual income, and savings have much significance in the Investment Decision Making Process, especially in the Indian context, it assumes greater significance. A study has been undertaken in Pune City of Maharashtra state, India to find its significance and the outcome of the study is narrated in the foregoing paragraphs.

\section{REVIEW OF LITERATURE}

To get more insight about investment pattern, researchers studied relational studies of investment pattern and demographic variables like Age, gender, income, educational background and occupation are studied.

\section{Age and Investment Pattern}

Age is found to be the most important determinants of investor style. Several researchers found the association between life cycle stages and investment pattern (Rajarajan 1994) found that the size of investment in the financial asset provides significant insight in the likely preference of individuals for particular class of financial instruments and investment approaches. The percentage of risky assets to total financial investment declined as the investor moves up through various stages in life cycle and Investment size below Rs. 50000 constitute the majority in all stages of life cycle. It can be said that the association of investment size and investors stage in life cycle does have a specific pattern. Similarly (Rajarajan 1999) concluded that the size of investment in financial assets and the percentage of risky assets in financial investment declines as the investor move up through the various stages in the life cycle.

Some researchers explored that investment choices differ according to age groups. Young investors find investing in equity shares/derivatives more comfortable, while old investors prefer PPF as their first choice. Middle aged investors prefer investing in mutual funds and NSC. (Meenu Verma 2008). Young aged investors (26-35) invest in mutual fund, while middle-aged investors (36-45) invest in debentures/bond, (Manish Mittal and R K Vyas, 2007). (Avinash Kumar Singh 2006) found that all the age groups give more important to invest in equity and except people those who are above 50 give important to insurance, fixed deposits and tax saving benefits. The studies relating to age and specific investment avenue (Karthikeyan 2001) has conducted research on Small Investors Perception on Post office saving Schemes and found that there was significant 
difference among the four age groups, in the level of awareness for Kisan Vikas Patra (KVP), National Savings Scheme (NSS), and deposit Scheme for retired Employees (DSRE), and the overall scores are confirmed that the level of awareness among investors in the old age group was higher than in those of young age group. Thus it can be concluded that that as age increases, the ability to take risks decreases and people go towards safer investments,

On the contrary, (Gnana Desigan C, S. Kalaiselvi and L. Anusuya 2006) concluded that age of the women investors and level of awareness about investment is not associated. (P. Vinoth Raj 2012) found that there is a strong negative correlation between Age and Risk tolerance level of the investor.

Above literature reveals that life cycle characteristic as segmentation variable provides an opportunity for segmentation of investors and blurs some differences between individual investors and their financial service need.

\section{Gender and Investment Pattern}

Traditionally men were the target segment of financial institutions, while women were viewed as feeling much less confident with financial services. But recent societal developments e.g., the demise of the nuclear family, the career-seeking woman have made women more knowledgeable of financial services.

Many researchers focused on investment preferences as per gender. (Manish Mittal and R K Vyas, 2007) found that males and females differ significantly in their choice of investment. Females prefer bank /postoffice deposits and least prefers equity shares and vice versa with male. Similarly, females prefer bank FD, insurance and bullions, (Meenu Verma 2008). The difference however is insignificant with medium risk - medium return investments as debentures, mutual funds and real estate/bullions. Similarly Meenu Verma (2008) noted that males prefer real estate, PPF and equity shares as attractive avenues for investment, (Karthikeyan 2001) has found that there are no differences were observed among male and female investors except for investment avenues such as NSS and KVP.

According to risk bearing capacity in investment decision making, (Manish Mittal and Dr. R. K. Vyas 2009) (Crosnan and Gneezy 2004) concluded that women are more risk averse and prefer low risk fixed income investments. Similarly, Eckel and Grossmann (2001) found significant gender differences in choices between several risky prospects with women indicating a preference for the less risky prospect. On the other hand, although Schubert et.al. (1999) found gender differences in abstract gambling decisions, the differences disappeared with the introduction of an investment decision context. Kruse and Thompson (2003) also found no significant differences between men and women in low probability loss situations. For choices under ambiguity, Powell and Ansic (1997) found that women are more uncertainty averse than men irrespective of familiarity, framing or costs. In their experiment, individuals demonstrate ambiguity adverse behavior in unfamiliar situations when compared to familiar ones. Schubert etal. (2000) found weak differences under two different formats of ambiguity but again no differences under risk. Giridhari Mohanta and Dr. Sathya Swaroop Debasish (2011) observed that there is significant role of income and occupation in investment avenue selection by the male and female investors. Mostly male investors are found as active participant in avenue selection than female and generally they 
are sound in these two respects than female investors. Also (Srinivasan Sakthi K, Lakshmi Devi S 2006) concluded that there is significant relationship between gender and percentage of income saved by the respondents.

Therefore it is evidence that women are more risk averse then men in general and this translates to investing in less risky assets in their investment plans. Differences in financial literacy between men and women also explain differences in their investment decisions. It reveals that gender-based segmentation is always useful for financial services marketers in order to adapt their communications policy to the degree to which the genders understand the complex nature of financial services.

\section{Income and Investment}

Income is very important determinant of investment decision making. Investment pattern changes according to different income group such as lower, middle and higher income group. Every group is having different savings and different investment preferences. Respondents from lower income groups i.e less than Rs. 1 lakh per annum invest in low-risk investments like post office deposits, Manish Mittal and R K Vyas, (2007), Income group less than 2.5 lakhs prefer NSC Meenu Verma(2008), post office savings and bank deposits (Srivastava Aman, 2007).

Investors with Middle income groups i.e. Rs. 1 lakh-2.5 lakhs invest in moderate investment avenues such as mutual funds, (Manish Mittal and R K Vyas, 2007). Bank FD and mutual funds. Meenu Verma(2008).

People from high income group with income between Rs. 2.5-4 lakhs invest in equities, Manish Mittal and R K Vyas, (2007) Real estate, (Meenu Verma, 2008),Stock and Real Estate (Srivastava Aman, 2007).

While studying association between investment avenues and Income level, (N. Geetha, Dr. M. Ramesh 2011) Also, (Srinivasan Sakthi K 2006) found that Income and percentage of income saved are significantly related and income and purpose of savings are insignificantly related.

On the contrary, Suman and D.P.Warne (2012) stated that the annual income and the annual saving are given importance of consideration by the respondents, because the level of income decides the level of savings.

Above studies reveal that the investment preferences are different among various income groups. As the income rises, the proportion of investment arises. Very few studies have been conducted in this context. Therefore preferred Investment Avenues and income group segmentation can be widened to get more insight in financial service industry.

\section{Education and Investment}

Education is also playing vital role in making choice of an investment. Manish Mittal and R K Vyas, (2007) found that Investors with less education prefer high-risk investments, such as, equity and derivatives. Undergraduate investors invest in high risk, high-return investments, such as, derivatives and real estate/bullion. Graduates prefer moderate risk and moderate return investments like debentures/bonds, while postgraduates and professionals invest in mutual funds and equity. They concluded that the propensity to take risk decreases with increase in education 
level. Also, (Bhagaban Das,. Sangeeta Mohanty, Nikhil Chandra Shil (2008) observed that investors with the graduate and postgraduate level of academic qualification are investing more in life insurance and the professionals are investing more in mutual fund.

While studying association between education level and investment avenues, (N. Geetha, M. Ramesh 2011) and (K.C.John, Sasi Kumar; P.Vikkraman. 2011) observed insignificant relation between education and investment avenues. Similarly, (Gnana Desigan C, S. Kalaiselvi and L. Anusuya 2006) found insignificant association between educational level and level of awareness about investment. On the contrary (Joseph Anbarasu D, Clifford Paul S, and Annette B 2011) observed a strong relationship between educational qualification and the opinion that saving is important.

Above literature reveals that very few studies have been made in relation to education and investment. Therefore it is difficult to depict exact trend of association between education and investment pattern.

\section{Occupation and Investment Pattern}

Individuals belonging to different occupations exhibit varying investment pattern. Every occupation is having different income. Some occupations are having fixed income and vice versa. Accordingly investment choices are different. Generally Service class, Profession, Businessman, Students and retired persons are the major classes under the head of occupation.

Service class people invest their savings in equities and Mutual Fund (Mittal Manish, 2007), PPF and Post Office schemes (Verma, 2008).

Business class prefer to invest in debentures, bonds, real estate and bullions (Mittal Manish, 2007), real estate and bullions (Verma, 2008),

Professionals invest in post office schemes and derivatives (Mittal Manish, 2007), Mutual Funds and insurance (Verma, 2008).

Housewives prefer safe investments like real estate, bullions (Mittal Manish, 2007), bank FD's and bullions (Verma, 2008) Students choose high risk investment like derivatives and equities (Mittal Manish, 2007) and equity and MF (Verma, 2008).

Government servants invest more in life insurance, (Bhagaban Das, Sangeeta Mohanty, Nikhil Chandra Shil 2008)

Private sector employees invest in Mutual funds, (Bhagaban Das, Sangeeta Mohanty, Nikhil Chandra 2008),

Farmers invest in Real Estate 44.90\%, 19.85\% in Deposits, 16.36\% in Gold, 9.76\% in Movable Property, 6.21\% in Business Assets, $2.73 \%$ in LIC and 0.20\% in Mutual Funds. And lastly Retired persons prefer to invest in PO and PPF (Verma, 2008), 57.78\% in Real Estate, 19.17\% in deposits, $12.06 \%$ in Gold, $4.90 \%$ in movable property, $4.18 \%$ in Financial Securities, $1.14 \%$ in Mutual Funds, $0.77 \%$ in LIC.

While studying association between Occupation and investment, (Joseph Anbarasu D, Clifford Paul S, and Annette B (2011) found that there is strong relationship between occupation of the respondents and the amount saved. (Gnana Desigan C, S. Kalaiselvi and L. Anusuya (2006) concluded that there is Significant association between occupation and level of awareness about 
investment. K.C.John Sasi Kumar; Dr.P.Vikkraman. (2011) found that there is a significant difference among occupation of investors.

Above studies reveal that occupation of investors play important role in investment decision making. Investment choices on the basis of occupation are mainly associated with risk bearing capacity of investors.

From the above literature, demographic variables are one of the major determinants which influence investment decision making of investors. Demographic factors, apart from other factors, exhibit the major characteristics of individual investors. Investment decisions differ from individual to individual who in turn differ demographically. The study is to find whether the demographic factors, to what extent, such as gender, age, education, occupation, and income, have influence over several elements of investment decisions.

\section{RESEARCH METHODOLOGY}

Present study is based on Empirical Research. Present research work is set to test following hypotheses.

Ho1 - There is no significant difference into investment pattern of individual investor on the magnitude of demographic profile. The demographic profile includes Age, Gender, Occupation, Income, Educational qualification.

Structured Schedule was used to collect primary data. It was divided into five parts. The structures were Percentage of existing investment and future preferences, Objectives behind Investment, Guiding Factors, Sources of information availed and demographic profile of sample respondents. The scope of the research was the metropolitan city of Pune. Stratified convenient sampling technique was used to draw sample from population. Stratification is done on the basis Socioeconomic Classes. These Socio-economic Classes are defined on the basis of number of household items owned and educational qualification. Samples from all groups were approached conveniently. Total sample Size was 670. Collected data are classified using electronic spread sheet; various statistical tools like ANOVA, Mann Whiteny 'U' test, Kruskal- Wallis test are used to analyze the data.

\section{DATA ANALYSIS AND DISCUSSIONS}

Above stated hypotheses are tested with respect to existing investment made by samples in respective investment instruments. All stated investment instruments are taken for testing of hypotheses.

\section{Investment Instrument Wise hypotheses testing} Hypothesis Testing for Gender and Investment pattern

Hypothesis related to Gender is tested with the help of Independent sample ' $t$ ' test

Following table shows independent sample ' $t$ ' testing of existing investment in investment instruments on the basis of Gender 


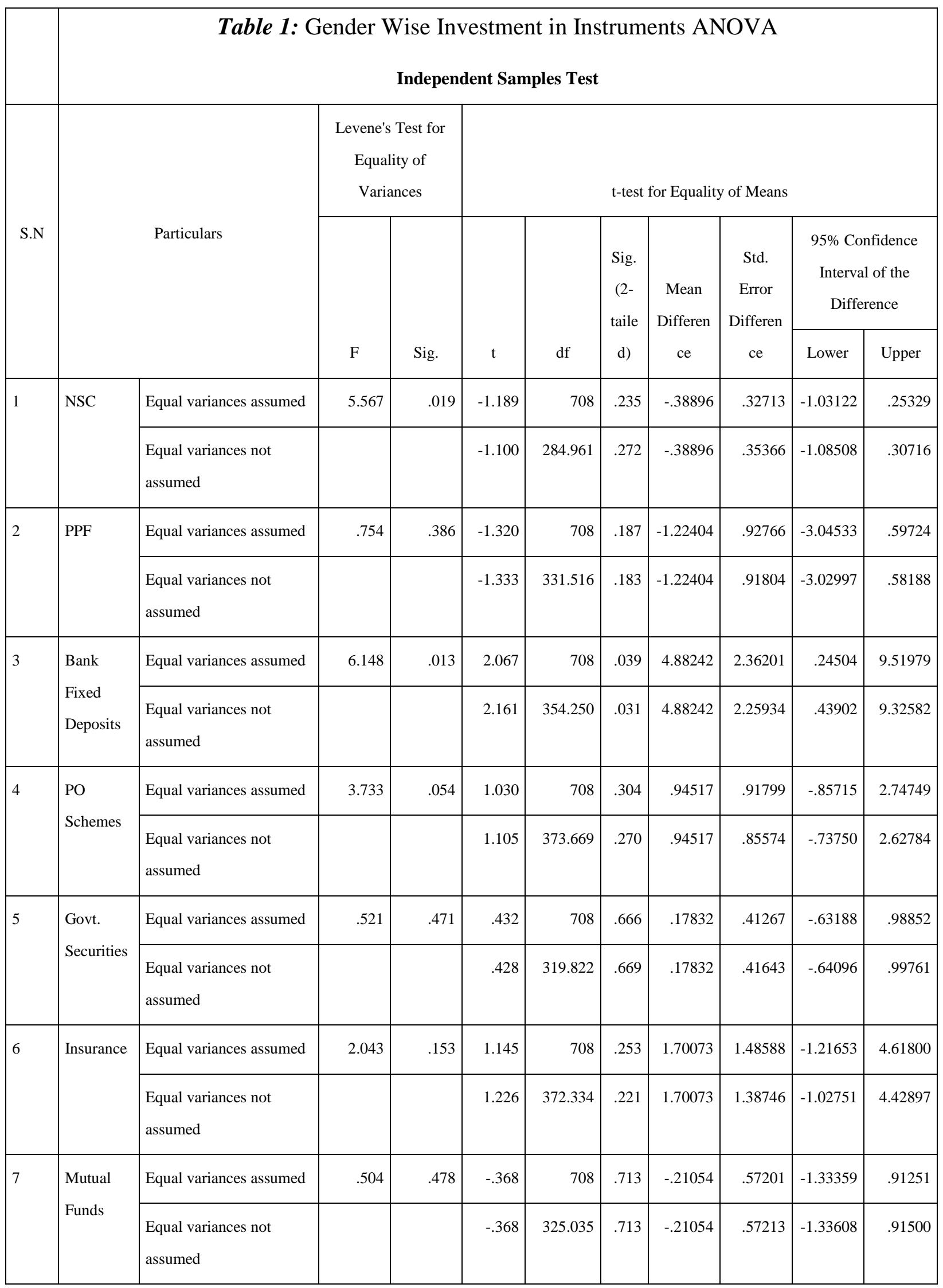


Impact Factor: 2.035 (I2OR)

\begin{tabular}{|c|c|c|c|c|c|c|c|c|c|c|c|}
\hline \multirow[t]{2}{*}{8} & \multirow[t]{2}{*}{ ELSS } & Equal variances assumed & 1.690 & .194 & -.645 & 708 & .519 & -.10917 & .16932 & -.44159 & .22325 \\
\hline & & $\begin{array}{l}\text { Equal variances not } \\
\text { assumed }\end{array}$ & & & -.555 & 256.987 & .580 & -.10917 & .19678 & -.49667 & .27833 \\
\hline \multirow[t]{2}{*}{9} & \multirow{2}{*}{$\begin{array}{l}\text { Debenture } \\
\mathrm{s}\end{array}$} & Equal variances assumed & 7.115 & .008 & -1.284 & 708 & .200 & -.31725 & .24707 & -.80232 & .16783 \\
\hline & & $\begin{array}{l}\text { Equal variances not } \\
\text { assumed }\end{array}$ & & & -.942 & 215.621 & .347 & -.31725 & .33670 & -.98089 & .34639 \\
\hline \multirow[t]{2}{*}{10} & \multirow[t]{2}{*}{ Bonds } & Equal variances assumed & 3.110 & .078 & -.969 & 708 & .333 & -.31345 & .32361 & -.94880 & .32190 \\
\hline & & $\begin{array}{l}\text { Equal variances not } \\
\text { assumed }\end{array}$ & & & -.939 & 307.594 & .349 & -.31345 & .33387 & -.97040 & .34350 \\
\hline \multirow[t]{2}{*}{11} & \multirow[t]{2}{*}{ Gold } & Equal variances assumed & 20.121 & .000 & -2.831 & 708 & .005 & -2.74245 & .96867 & -4.64425 & -.84065 \\
\hline & & $\begin{array}{l}\text { Equal variances not } \\
\text { assumed }\end{array}$ & & & -2.580 & 278.575 & .010 & -2.74245 & 1.06294 & -4.83486 & -.65004 \\
\hline \multirow[t]{2}{*}{12} & \multirow{2}{*}{$\begin{array}{l}\text { Company } \\
\text { Deposits }\end{array}$} & Equal variances assumed & 13.707 & .000 & 1.839 & 708 & .066 & .40076 & .21793 & -.02710 & .82863 \\
\hline & & $\begin{array}{l}\text { Equal variances not } \\
\text { assumed }\end{array}$ & & & 3.088 & 523.000 & .002 & .40076 & .12978 & .14581 & .65572 \\
\hline \multirow[t]{2}{*}{13} & \multirow[t]{2}{*}{ SIP } & Equal variances assumed & .016 & .901 & -.070 & 708 & .944 & -.04586 & .65210 & -1.32614 & 1.23441 \\
\hline & & $\begin{array}{l}\text { Equal variances not } \\
\text { assumed }\end{array}$ & & & -.082 & 446.514 & .935 & -.04586 & .56197 & -1.15030 & 1.05857 \\
\hline \multirow[t]{2}{*}{14} & \multirow[t]{2}{*}{ ULIP } & Equal variances assumed & 20.959 & .000 & -2.297 & 708 & .022 & -.57560 & .25058 & -1.06756 & -.08363 \\
\hline & & $\begin{array}{l}\text { Equal variances not } \\
\text { assumed }\end{array}$ & & & -1.906 & 245.460 & .058 & -.57560 & .30201 & -1.17045 & .01926 \\
\hline \multirow[t]{2}{*}{15} & \multirow{2}{*}{$\begin{array}{l}\text { Commodi } \\
\text { ty }\end{array}$} & Equal variances assumed & 38.456 & .000 & 2.963 & 708 & .003 & .70005 & .23630 & .23613 & 1.16398 \\
\hline & & $\begin{array}{l}\text { Equal variances not } \\
\text { assumed }\end{array}$ & & & 4.685 & 649.503 & .000 & .70005 & .14943 & .40663 & .99348 \\
\hline \multirow[t]{2}{*}{16} & \multirow[t]{2}{*}{ NBFC } & Equal variances assumed & 2.864 & .091 & .843 & 708 & .400 & .03817 & .04528 & -.05072 & .12706 \\
\hline & & $\begin{array}{l}\text { Equal variances not } \\
\text { assumed }\end{array}$ & & & 1.416 & 523.000 & .157 & .03817 & .02696 & -.01480 & .09114 \\
\hline 17 & Livestock & Equal variances assumed & 3.736 & .054 & -.989 & 708 & .323 & -.72774 & .73618 & -2.17309 & .71762 \\
\hline
\end{tabular}




\begin{tabular}{|c|c|c|c|c|c|c|c|c|c|c|c|}
\hline & & $\begin{array}{l}\text { Equal variances not } \\
\text { assumed }\end{array}$ & & & -.928 & 291.594 & .354 & -.72774 & .78435 & -2.27145 & .81598 \\
\hline \multirow[t]{2}{*}{18} & \multirow{2}{*}{$\begin{array}{l}\text { Real } \\
\text { Estate }\end{array}$} & Equal variances assumed & 10.389 & .001 & 1.777 & 708 & .076 & 1.18175 & .66515 & -.12415 & 2.48764 \\
\hline & & $\begin{array}{l}\text { Equal variances not } \\
\text { assumed }\end{array}$ & & & 1.775 & 324.534 & .077 & 1.18175 & .66584 & -.12815 & 2.49165 \\
\hline \multirow[t]{2}{*}{19} & \multirow{2}{*}{$\begin{array}{l}\text { Chit } \\
\text { Funds }\end{array}$} & Equal variances assumed & 48.018 & .000 & -3.865 & 708 & .000 & -5.15688 & 1.33412 & -7.77619 & -2.53757 \\
\hline & & $\begin{array}{l}\text { Equal variances not } \\
\text { assumed }\end{array}$ & & & -2.952 & 223.992 & .003 & -5.15688 & 1.74719 & -8.59991 & -1.71385 \\
\hline \multirow[t]{2}{*}{20} & \multirow[t]{2}{*}{ Shares } & Equal variances assumed & 11.325 & .001 & 2.060 & 708 & .040 & 1.31772 & .63982 & .06155 & 2.57389 \\
\hline & & $\begin{array}{l}\text { Equal variances not } \\
\text { assumed }\end{array}$ & & & 2.129 & 346.476 & .034 & 1.31772 & 61885 & .10054 & 2.53490 \\
\hline \multirow[t]{2}{*}{21} & \multirow{2}{*}{$\begin{array}{l}\text { Forex } \\
\text { Market }\end{array}$} & Equal variances assumed & 2.359 & .125 & .764 & 708 & .445 & .10845 & .14203 & -.17041 & .38731 \\
\hline & & $\begin{array}{l}\text { Equal variances not } \\
\text { assumed }\end{array}$ & & & 1.102 & 702.948 & .271 & .10845 & .09840 & -.08474 & .30164 \\
\hline \multirow[t]{2}{*}{22} & \multirow{2}{*}{$\begin{array}{l}\text { Private } \\
\text { Equity } \\
\text { Investmen } \\
\text { ts }\end{array}$} & Equal variances assumed & 6.635 & .010 & 1.278 & 708 & .202 & .07634 & .05975 & -.04097 & .19364 \\
\hline & & $\begin{array}{l}\text { Equal variances not } \\
\text { assumed }\end{array}$ & & & 2.145 & 523.000 & .032 & .07634 & .03558 & .00644 & .14623 \\
\hline \multirow[t]{2}{*}{23} & \multirow{2}{*}{$\begin{array}{l}\text { Credit } \\
\text { Society }\end{array}$} & Equal variances assumed & 2.534 & .112 & -.849 & 708 & .396 & -1.00581 & 1.18491 & -3.33217 & 1.32056 \\
\hline & & $\begin{array}{l}\text { Equal variances not } \\
\text { assumed }\end{array}$ & & & -.760 & 271.186 & .448 & -1.00581 & 1.32427 & -3.61296 & 1.60135 \\
\hline \multirow[t]{2}{*}{24} & \multirow{2}{*}{$\begin{array}{l}\text { Any } \\
\text { Others }\end{array}$} & Equal variances assumed & 7.176 & .008 & 1.390 & 708 & .165 & 1.28786 & .92639 & -.53093 & 3.10665 \\
\hline & & $\begin{array}{l}\text { Equal variances not } \\
\text { assumed }\end{array}$ & & & 1.899 & 654.753 & .058 & 1.28786 & .67807 & -.04358 & 2.61930 \\
\hline
\end{tabular}

\section{Source: (Compiled by Researcher)}

The table of comparison by 't' test reveals that there is significant difference into investment instruments viz. NSC, Bank Deposits, Debentures, Gold, Company Deposits, ULIP, Commodity Market, Real Estate, Chit Funds, Shares, Private Equity Investments, Others The ' $t$ ' is significant at $95 \%$ confidence level.

The test results are also cross checked with the help of Mann-Whitney 'U' test. Following table shows Mann-Whitney ' $U$ ' test between Investment Instruments 
Table 2: Mann-Whitney 'U' Test between Investment Instruments

\begin{tabular}{|l|l|l|l|l|l|}
\hline S.N & Investment Instruments & $\begin{array}{l}\text { Mann-Whitney } \\
\text { 'U' }\end{array}$ & $\begin{array}{l}\text { Wilcoxon } \\
\text { W }\end{array}$ & Z & $\begin{array}{l}\text { Asymp. Sig. } \\
\text { (2-tailed) }\end{array}$ \\
\hline 1 & NSC & 47466 & 185016 & -1.01 & 0.31 \\
\hline 2 & PPF & 44861 & 182411 & -1.87 & 0.06 \\
\hline 3 & Bank Fixed Deposits & 44782 & 62173 & -1.65 & 0.10 \\
\hline 4 & PO Schemes & 47359.5 & 64750.5 & -0.79 & 0.43 \\
\hline 5 & Government Securities & 47585.5 & 64976.5 & -0.88 & 0.38 \\
\hline 6 & Insurance & 46536 & 63927 & -0.94 & 0.35 \\
\hline 7 & Mutual Funds & 48057.5 & 185607.5 & -0.40 & 0.69 \\
\hline 8 & Cash in Hand & 48732 & 66123 & 0.00 & 1.00 \\
\hline 9 & ELSS & 48534 & 186084 & -0.31 & 0.76 \\
\hline 10 & Debentures & 48402.5 & 65793.5 & -0.45 & 0.65 \\
\hline 11 & Bonds & 47052 & 184602 & -1.20 & 0.23 \\
\hline 12 & Gold/ Silver & 43811.5 & 181361.5 & -2.49 & 0.01 \\
\hline 13 & Company Deposits & 47337 & 64728 & -2.33 & 0.02 \\
\hline 14 & SIP & 47547.5 & 185097.5 & -0.73 & 0.47 \\
\hline 15 & ULIP & 46818 & 184368 & -1.97 & 0.05 \\
\hline 16 & Commodity Market & 46196 & 63587 & -2.98 & 0.00 \\
\hline 17 & NBFC Schemes & 48546 & 65937 & -0.84 & 0.40 \\
\hline 18 & Live Stock & 47532.5 & 185082.5 & -0.75 & 0.45 \\
\hline 19 & Real Estate & 45010.5 & 62401.5 & -2.59 & 0.01 \\
\hline 20 & Chit Funds & 44030 & 181580 & -3.63 & 0.00 \\
\hline 21 & Shares & 43967 & 61358 & -2.73 & 0.01 \\
\hline 22 & Forex Market & 48620 & 66011 & -0.32 & 0.75 \\
\hline & Private Equity & 48267 & 65658 & -1.34 & 0.18 \\
\hline 23 & Investments & & & & \\
\hline 24 & Credit Society & 47721.5 & 185271.5 & -0.95 & 0.34 \\
\hline 25 & Any Other & 48033 & 65424 & -0.53 & 0.59 \\
\hline
\end{tabular}

Source: (Compiled by Researcher)

Table 2 shows that the Mann - Whitney ' $U$ ' test is significant reveals that there is significant difference into investment instruments viz. Gold/Silver, Company Deposits, ULIP, Commodity Market, Real Estate, Chit Funds and Shares.

Independent sample ' $t$ ' test and Mann - Whitney ' $U$ ' test shows that null hypotheses is rejected with investment instruments viz. Gold/Silver, Company Deposits, ULIP, Commodity Market, Real Estate, Chit Funds and Shares. It means that alternative hypotheses is accepted that there is significant difference into investment pattern on the basis of Gender.

Following table shows ANOVA testing of the investment in instruments as per age group of sample investors.

Hypothesis Testing for Age Group and Investment pattern

Hypothesis related to Age groups is tested with the help of ANOVA

Following table shows ANOVA table for existing investment in investment instruments on the basis of Age group 


\begin{tabular}{|c|c|c|c|c|c|c|c|}
\hline \multicolumn{8}{|c|}{ Table 3: Age Group wise Investment in Instruments ANOVA } \\
\hline S.N & & & Sum of Squares & df & Mean Square & $\mathrm{F}$ & Sig. \\
\hline \multirow{3}{*}{1} & \multirow{3}{*}{$\mathrm{NSC}$} & Between Groups & 258.503 & 8 & 32.313 & 2.229 & .024 \\
\hline & & Within Groups & 10162.659 & 701 & 14.497 & & \\
\hline & & Total & 10421.162 & 709 & & & \\
\hline \multirow{3}{*}{2} & \multirow{3}{*}{ PPF } & Between Groups & 3821.865 & 8 & 477.733 & 4.185 & .000 \\
\hline & & Within Groups & 80019.684 & 701 & 114.151 & & \\
\hline & & Total & 83841.549 & 709 & & & \\
\hline \multirow{3}{*}{3} & \multirow{3}{*}{$\begin{array}{c}\text { Bank Fixed } \\
\text { Deposits }\end{array}$} & Between Groups & 16152.755 & 8 & 2019.094 & 2.674 & .007 \\
\hline & & Within Groups & 529346.400 & 701 & 755.130 & & \\
\hline & & Total & 545499.155 & 709 & & & \\
\hline \multirow{3}{*}{4} & \multirow{3}{*}{ PO Schemes } & Between Groups & 5159.073 & 8 & 644.884 & 5.881 & .000 \\
\hline & & Within Groups & 76866.420 & 701 & 109.653 & & \\
\hline & & Total & 82025.493 & 709 & & & \\
\hline \multirow{3}{*}{5} & \multirow{3}{*}{ Govt. Securities } & Between Groups & 74.053 & 8 & 9.257 & .394 & .924 \\
\hline & & Within Groups & 16481.193 & 701 & 23.511 & & \\
\hline & & Total & 16555.246 & 709 & & & \\
\hline \multirow{3}{*}{6} & \multirow{3}{*}{ Insurance } & Between Groups & 10624.096 & 8 & 1328.012 & 4.556 & .000 \\
\hline & & Within Groups & 204352.699 & 701 & 291.516 & & \\
\hline & & Total & 214976.796 & 709 & & & \\
\hline \multirow{3}{*}{7} & \multirow{3}{*}{ Mutual Funds } & Between Groups & 928.753 & 8 & 116.094 & 2.636 & .008 \\
\hline & & Within Groups & 30877.726 & 701 & 44.048 & & \\
\hline & & Total & 31806.479 & 709 & & & \\
\hline \multirow{2}{*}{8} & \multirow{2}{*}{ Cash } & Between Groups & .000 & 8 & .000 & & \\
\hline & & Within Groups & .000 & 701 & .000 & & \\
\hline
\end{tabular}




\begin{tabular}{|c|c|c|c|c|c|c|c|}
\hline & & Total & .000 & 709 & & & \\
\hline \multirow{3}{*}{9} & \multirow{3}{*}{ ELSS } & Between Groups & 28.161 & 8 & 3.520 & .894 & .521 \\
\hline & & Within Groups & 2759.726 & 701 & 3.937 & & \\
\hline & & Total & 2787.887 & 709 & & & \\
\hline \multirow{3}{*}{10} & \multirow{3}{*}{ Debentures } & Between Groups & 52.415 & 8 & 6.552 & .779 & .621 \\
\hline & & Within Groups & 5894.205 & 701 & 8.408 & & \\
\hline & & Total & 5946.620 & 709 & & & \\
\hline \multirow{3}{*}{11} & \multirow{3}{*}{ Bonds } & Between Groups & 450.330 & 8 & 56.291 & 4.051 & .000 \\
\hline & & Within Groups & 9741.219 & 701 & 13.896 & & \\
\hline & & Total & 10191.549 & 709 & & & \\
\hline \multirow{3}{*}{12} & \multirow{3}{*}{ Gold/Silver } & Between Groups & 10163.340 & 8 & 1270.417 & 10.852 & .000 \\
\hline & & Within Groups & 82063.456 & 701 & 117.066 & & \\
\hline & & Total & 92226.796 & 709 & & & \\
\hline \multirow{3}{*}{13} & \multirow{3}{*}{$\begin{array}{l}\text { Company } \\
\text { Deposits }\end{array}$} & Between Groups & 70.363 & 8 & 8.795 & 1.350 & .216 \\
\hline & & Within Groups & 4567.524 & 701 & 6.516 & & \\
\hline & & Total & 4637.887 & 709 & & & \\
\hline \multirow{3}{*}{14} & \multirow{3}{*}{ SIP } & Between Groups & 1977.209 & 8 & 247.151 & 4.403 & .000 \\
\hline & & Within Groups & 39351.101 & 701 & 56.136 & & \\
\hline & & Total & 41328.310 & 709 & & & \\
\hline \multirow{3}{*}{15} & \multirow{3}{*}{ ULIP } & Between Groups & 234.921 & 8 & 29.365 & 3.481 & .001 \\
\hline & & Within Groups & 5912.967 & 701 & 8.435 & & \\
\hline & & Total & 6147.887 & 709 & & & \\
\hline \multirow{3}{*}{16} & \multirow{3}{*}{$\begin{array}{c}\text { Commodity } \\
\text { Market }\end{array}$} & Between Groups & 100.847 & 8 & 12.606 & 1.639 & .110 \\
\hline & & Within Groups & 5393.132 & 701 & 7.693 & & \\
\hline & & Total & 5493.979 & 709 & & & \\
\hline 17 & NBFC Schemes & Between Groups & 2.537 & 8 & .317 & 1.129 & .341 \\
\hline
\end{tabular}




\begin{tabular}{|c|c|c|c|c|c|c|c|}
\hline & & Within Groups & 196.899 & 701 & .281 & & \\
\hline & & Total & 199.437 & 709 & & & \\
\hline \multirow{3}{*}{18} & \multirow{3}{*}{ Live Stock } & Between Groups & 4253.647 & 8 & 531.706 & 7.686 & .000 \\
\hline & & Within Groups & 48491.932 & 701 & 69.175 & & \\
\hline & & Total & 52745.579 & 709 & & & \\
\hline \multirow{3}{*}{19} & \multirow{3}{*}{ Real Estate } & Between Groups & 1024.705 & 8 & 128.088 & 2.129 & .031 \\
\hline & & Within Groups & 42165.486 & 701 & 60.150 & & \\
\hline & & Total & 43190.192 & 709 & & & \\
\hline \multirow{3}{*}{20} & \multirow{3}{*}{ Chit Funds } & Between Groups & 4036.097 & 8 & 504.512 & 2.049 & .039 \\
\hline & & Within Groups & 172600.382 & 701 & 246.220 & & \\
\hline & & Total & 176636.479 & 709 & & & \\
\hline \multirow{3}{*}{21} & \multirow{3}{*}{ Shares } & Between Groups & 1492.082 & 8 & 186.510 & 3.393 & .001 \\
\hline & & Within Groups & 38532.707 & 701 & 54.968 & & \\
\hline & & Total & 40024.789 & 709 & & & \\
\hline \multirow{3}{*}{22} & \multirow{3}{*}{ Forex Market } & Between Groups & 28.951 & 8 & 3.619 & 1.312 & .234 \\
\hline & & Within Groups & 1933.338 & 701 & 2.758 & & \\
\hline & & Total & 1962.289 & 709 & & & \\
\hline \multirow{3}{*}{23} & \multirow{3}{*}{$\begin{array}{l}\text { Private Equity } \\
\text { Investments }\end{array}$} & Between Groups & 5.834 & 8 & .729 & 1.495 & .155 \\
\hline & & Within Groups & 341.913 & 701 & .488 & & \\
\hline & & Total & 347.746 & 709 & & & \\
\hline \multirow{3}{*}{24} & \multirow{3}{*}{ Credit Society } & Between Groups & 8795.771 & 8 & 1099.471 & 6.031 & .000 \\
\hline & & Within Groups & 127798.913 & 701 & 182.309 & & \\
\hline & & Total & 136594.683 & 709 & & & \\
\hline \multirow{3}{*}{25} & \multirow{3}{*}{ Any Others } & Between Groups & 1773.080 & 8 & 221.635 & 1.898 & .058 \\
\hline & & Within Groups & 81861.567 & 701 & 116.778 & & \\
\hline & & Total & 83634.648 & 709 & & & \\
\hline
\end{tabular}


Source: (Compiled by Researcher)

Table 3 shows that ANOVA model is significant with investment instruments namely NSC, PPF, Bank Deposits, PO Schemes, Insurance, Mutual Funds, Bonds, Gold/Silver, SIP, ULIP, Live Stock, Real Estate, Chit Funds, Shares, and Credit Society. These instruments are significant at 95\% confidence level.

To verify the results of ANOVA, Kruskal Wallis Test is also performed.

Table: 4: Kruskal Wallis Test between Investment Instruments

\begin{tabular}{|c|c|c|c|c|}
\hline S.N & Investment Avenues & Chi-Square & $\mathrm{df}$ & Asymp. Sig. \\
\hline 1 & NSC & 43.36 & 6 & 0.00 \\
\hline 2 & PPF & 50.16 & 6 & 0.00 \\
\hline 3 & Bank Fixed Deposits & 135.92 & 6 & 0.00 \\
\hline 4 & PO Schemes & 102.24 & 6 & 0.00 \\
\hline 5 & Government Securities & 30.44 & 6 & 0.00 \\
\hline 6 & Insurance & 72.27 & 6 & 0.00 \\
\hline 7 & Mutual Funds & 130.89 & 6 & 0.00 \\
\hline 8 & ELSS & 35.60 & 6 & 0.00 \\
\hline 9 & Debentures & 13.23 & 6 & 0.04 \\
\hline 10 & Bonds & 93.64 & 6 & 0.00 \\
\hline 11 & Gold/ Silver & 82.74 & 6 & 0.00 \\
\hline 12 & Company Deposits & 15.02 & 6 & 0.02 \\
\hline 13 & SIP & 65.99 & 6 & 0.00 \\
\hline 14 & ULIP & 43.11 & 6 & 0.00 \\
\hline 15 & Commodity Market & 48.18 & 6 & 0.00 \\
\hline 16 & NBFC Schemes & 13.80 & 6 & 0.03 \\
\hline 17 & Live Stock & 54.09 & 6 & 0.00 \\
\hline 18 & Real Estate & 44.49 & 6 & 0.00 \\
\hline 19 & Chit Funds & 19.42 & 6 & 0.00 \\
\hline 20 & Shares & 97.82 & 6 & 0.00 \\
\hline 21 & Forex Market & 7.92 & 6 & 0.24 \\
\hline 22 & Private Equity & 1.92 & 6 & 0.93 \\
\hline 23 & Credit Society & 55.09 & 6 & 0.00 \\
\hline 24 & Any Other & 15.82 & 6 & 0.01 \\
\hline
\end{tabular}


Source: (Compiled by Researcher)

Table 4 shows that Kruskal Wallis test is significant with investment instruments namely NSC, PPF, Bank Deposits, PO Schemes, Government Securities, Insurance, Mutual Funds, Debentures, Bonds, Gold/Silver, Company Deposits, SIP, ULIP, Commodity Market, NBFC Schemes, Live Stock, Real Estate, Chit Funds, Shares, and Credit Society. These instruments are significant at 95\% confidence level.

It means that the results of ANOVA and Kruskal Wallis test shows that null hypotheses is rejected with investment instruments viz. NSC, PPF, Bank Deposits, PO Schemes, Insurance, Mutual Funds, Bonds, Gold/Silver, SIP, ULIP, Live Stock, Real Estate, Chit Funds, Shares, and Credit Society. It means that alternative hypotheses is accepted that there is significant difference into investment pattern on the basis of Age group of sample respondents.

Following table shows ANOVA testing of the investment in instruments as per Educational Qualification of sample investors.

\section{Hypothesis Testing for Educational Qualification and Investment pattern}

Hypothesis related to Educational Qualification is tested with the help of ANOVA

Following table shows ANOVA table for existing investment in investment instruments on the basis of Educational Qualification 


\begin{tabular}{|c|c|c|c|c|c|c|c|}
\hline \multicolumn{8}{|c|}{$\begin{array}{l}\text { Table 5: Educational Qualification wise Investment in Instruments ANOVA } \\
\qquad(\mathrm{n}=710)\end{array}$} \\
\hline S.N & \multicolumn{2}{|c|}{ Particulars } & Sum of & & Mean & & \\
\hline \multirow{3}{*}{1} & \multirow{3}{*}{ NSC } & Between Groups & 566.1 & 6 & 94.4 & 6.731 & . 000 \\
\hline & & Within Groups & 9855.0 & 703 & 14.0 & & \\
\hline & & Total & 10421.2 & 709 & & & \\
\hline \multirow{3}{*}{2} & \multirow{3}{*}{ PPF } & Between Groups & 4163.0 & 6 & 693.8 & 6.122 & .000 \\
\hline & & Within Groups & 79678.5 & 703 & 113.3 & & \\
\hline & & Total & 83841.5 & 709 & & & \\
\hline \multirow{3}{*}{3} & \multirow{3}{*}{$\begin{array}{c}\text { Bank Fixed } \\
\text { Deposits }\end{array}$} & Between Groups & 113584.6 & 6 & 18930.8 & 30.812 & .000 \\
\hline & & Within Groups & 431914.6 & 703 & 614.4 & & \\
\hline & & Total & 545499.2 & 709 & & & \\
\hline \multirow{3}{*}{4} & \multirow{3}{*}{ PO Schemes } & Between Groups & 11894.7 & 6 & 1982.5 & 19.872 & . 000 \\
\hline & & Within Groups & 70130.8 & 703 & 99.8 & & \\
\hline & & Total & 82025.5 & 709 & & & \\
\hline \multirow{3}{*}{5} & \multirow{3}{*}{$\begin{array}{c}\text { Govt. } \\
\text { Securities }\end{array}$} & Between Groups & 500.3 & 6 & 83.4 & 3.651 & .001 \\
\hline & & Within Groups & 16054.9 & 703 & 22.8 & & \\
\hline & & Total & 16555.2 & 709 & & & \\
\hline \multirow{3}{*}{6} & \multirow{3}{*}{ Insurance } & Between Groups & 8795.1 & 6 & 1465.9 & 4.998 & .000 \\
\hline & & Within Groups & 206181.7 & 703 & 293.3 & & \\
\hline & & Total & 214976.8 & 709 & & & \\
\hline \multirow{3}{*}{7} & \multirow{3}{*}{ Mutual Funds } & Between Groups & 4635.1 & 6 & 772.5 & 19.987 & .000 \\
\hline & & Within Groups & 27171.4 & 703 & 38.7 & & \\
\hline & & Total & 31806.5 & 709 & & & \\
\hline \multirow{2}{*}{8} & \multirow{2}{*}{ ELSS } & Between Groups & 111.0 & 6 & 18.5 & 4.861 & .000 \\
\hline & & Within Groups & 2676.8 & 703 & 3.8 & & \\
\hline
\end{tabular}




\begin{tabular}{|c|c|c|c|c|c|c|c|}
\hline & & Total & 2787.9 & 709 & & & \\
\hline \multirow{3}{*}{9} & \multirow{3}{*}{ Debentures } & Between Groups & 78.5 & 6 & 13.1 & 1.566 & .154 \\
\hline & & Within Groups & 5868.2 & 703 & 8.3 & & \\
\hline & & Total & 5946.6 & 709 & & & \\
\hline \multirow{3}{*}{10} & \multirow{3}{*}{ Bonds } & Between Groups & 1227.1 & 6 & 204.5 & 16.038 & .000 \\
\hline & & Within Groups & 8964.5 & 703 & 12.8 & & \\
\hline & & Total & 10191.5 & 709 & & & \\
\hline \multirow{3}{*}{11} & \multirow{3}{*}{ Gold/Silver } & Between Groups & 8449.8 & 6 & 1408.3 & 11.818 & .000 \\
\hline & & Within Groups & 83777.0 & 703 & 119.2 & & \\
\hline & & Total & 92226.8 & 709 & & & \\
\hline \multirow{3}{*}{12} & \multirow{3}{*}{$\begin{array}{l}\text { Company } \\
\text { Deposits }\end{array}$} & Between Groups & 60.3 & 6 & 10.0 & 1.543 & .161 \\
\hline & & Within Groups & 4577.6 & 703 & 6.5 & & \\
\hline & & Total & 4637.9 & 709 & & & \\
\hline \multirow{3}{*}{13} & \multirow{3}{*}{ SIP } & Between Groups & 1855.8 & 6 & 309.3 & 5.508 & .000 \\
\hline & & Within Groups & 39472.6 & 703 & 56.1 & & \\
\hline & & Total & 41328.3 & 709 & & & \\
\hline \multirow{3}{*}{14} & \multirow{3}{*}{ ULIP } & Between Groups & 339.4 & 6 & 56.6 & 6.846 & .000 \\
\hline & & Within Groups & 5808.5 & 703 & 8.3 & & \\
\hline & & Total & 6147.9 & 709 & & & \\
\hline \multirow{3}{*}{15} & \multirow{3}{*}{$\begin{array}{c}\text { Commodity } \\
\text { Market }\end{array}$} & Between Groups & 337.6 & 6 & 56.3 & 7.672 & .000 \\
\hline & & Within Groups & 5156.4 & 703 & 7.3 & & \\
\hline & & Total & 5494.0 & 709 & & & \\
\hline \multirow{3}{*}{16} & \multirow{3}{*}{$\begin{array}{c}\text { NBFC } \\
\text { Schemes }\end{array}$} & Between Groups & 3.9 & 6 & .6 & 2.325 & .031 \\
\hline & & Within Groups & 195.6 & 703 & .3 & & \\
\hline & & Total & 199.4 & 709 & & & \\
\hline 17 & Live Stock & Between Groups & 3041.9 & 6 & 507.0 & 7.171 & .000 \\
\hline
\end{tabular}




\begin{tabular}{|c|c|c|c|c|c|c|c|}
\hline & & Within Groups & 49703.6 & 703 & 70.7 & & \\
\hline & & Total & 52745.6 & 709 & & & \\
\hline \multirow{3}{*}{18} & \multirow{3}{*}{ Real Estate } & Between Groups & 1878.4 & 6 & 313.1 & 5.328 & .000 \\
\hline & & Within Groups & 41311.8 & 703 & 58.8 & & \\
\hline & & Total & 43190.2 & 709 & & & \\
\hline \multirow{3}{*}{19} & \multirow{3}{*}{ Chit Funds } & Between Groups & 3260.3 & 6 & 543.4 & 2.203 & .041 \\
\hline & & Within Groups & 173376.1 & 703 & 246.6 & & \\
\hline & & Total & 176636.5 & 709 & & & \\
\hline \multirow{3}{*}{20} & \multirow{3}{*}{ Shares } & Between Groups & 3980.1 & 6 & 663.3 & 12.938 & .000 \\
\hline & & Within Groups & 36044.7 & 703 & 51.3 & & \\
\hline & & Total & 40024.8 & 709 & & & \\
\hline \multirow{3}{*}{21} & \multirow{3}{*}{ Forex Market } & Between Groups & 22.9 & 6 & 3.8 & 1.385 & .218 \\
\hline & & Within Groups & 1939.4 & 703 & 2.8 & & \\
\hline & & Total & 1962.3 & 709 & & & \\
\hline \multirow{3}{*}{22} & \multirow{3}{*}{$\begin{array}{l}\text { Private Equity } \\
\text { Investments }\end{array}$} & Between Groups & 1.3 & 6 & .2 & .443 & .850 \\
\hline & & Within Groups & 346.4 & 703 & .5 & & \\
\hline & & Total & 347.7 & 709 & & & \\
\hline \multirow{3}{*}{23} & \multirow{3}{*}{ Credit Society } & Between Groups & 13289.4 & 6 & 2214.9 & 12.628 & .000 \\
\hline & & Within Groups & 123305.3 & 703 & 175.4 & & \\
\hline & & Total & 136594.7 & 709 & & & \\
\hline \multirow{3}{*}{24} & \multirow{3}{*}{ Any Others } & Between Groups & 1437.1 & 6 & 239.5 & 2.048 & .057 \\
\hline & & Within Groups & 82197.6 & 703 & 116.9 & & \\
\hline & & Total & 83634.6 & 709 & & & \\
\hline
\end{tabular}

Source: (Compiled by Researcher)

Table 5 shows that ANOVA model is significant with investment instruments namely NSC, PPF, Bank Deposits, PO Schemes, Insurance, Mutual Funds, ELSS, Bonds, Gold/Silver, SIP, ULIP, Commodity Market, NBFC Schemes, Live Stock, Real Estate, Chit Funds, Shares, and Credit Society. These instruments are significant at $95 \%$ confidence level. 
To verify the results of ANOVA, Kruskal Wallis Test is also performed

Table 6: Kruskal Wallis Test between Investment Instruments

\begin{tabular}{|c|c|c|c|c|}
\hline S.N & Investment Avenues & Chi-Square & Df & Asymp. Sig. \\
\hline 1 & NSC & 43.36 & 6 & 0.00 \\
\hline 2 & PPF & 50.16 & 6 & 0.00 \\
\hline 3 & Bank Fixed Deposits & 135.92 & 6 & 0.00 \\
\hline 4 & PO Schemes & 102.24 & 6 & 0.00 \\
\hline 5 & Government Securities & 30.44 & 6 & 0.00 \\
\hline 6 & Insurance & 72.27 & 6 & 0.00 \\
\hline 7 & Mutual Funds & 130.89 & 6 & 0.00 \\
\hline 8 & ELSS & 35.60 & 6 & 0.00 \\
\hline 9 & Debentures & 13.23 & 6 & 0.04 \\
\hline 10 & Bonds & 93.64 & 6 & 0.00 \\
\hline 11 & Gold/ Silver & 82.74 & 6 & 0.00 \\
\hline 12 & Company Deposits & 15.02 & 6 & 0.02 \\
\hline 13 & SIP & 65.99 & 6 & 0.00 \\
\hline 14 & ULIP & 43.11 & 6 & 0.00 \\
\hline 15 & Commodity Market & 48.18 & 6 & 0.00 \\
\hline 16 & NBFC Schemes & 13.80 & 6 & 0.03 \\
\hline 17 & Live Stock & 54.09 & 6 & 0.00 \\
\hline 18 & Real Estate & 44.49 & 6 & 0.00 \\
\hline 19 & Chit Funds & 19.42 & 6 & 0.00 \\
\hline 20 & Shares & 97.82 & 6 & 0.00 \\
\hline 21 & Forex Market & 7.92 & 6 & 0.24 \\
\hline 22 & Private Equity & 1.92 & 6 & 0.93 \\
\hline 23 & Credit Society & 55.09 & 6 & 0.00 \\
\hline 24 & Any Other & 15.82 & 6 & 0.01 \\
\hline
\end{tabular}

$(\mathrm{n}=710)$

Source: (Compiled by Researcher) 
Table 6 shows that Kruskal wallis is significant with investment instruments namely NSC, PPF, Bank Deposits, PO Schemes, Government Securities, Insurance, Mutual Funds, Debentures, Bonds, Gold/Silver, Company Deposits, SIP, ULIP, Commodity Market, NBFC Schemes, Live Stock, Real Estate, Chit Funds, Shares, and Credit Society. These instruments are significant at 95\% confidence level.

It means that the results of ANOVA and Kruskal Wallis test shows that null hypotheses is rejected with investment instruments viz. NSC, PPF, Bank Deposits, PO Schemes, Insurance, Mutual Funds, ELSS, Bonds, Gold/Silver, SIP, ULIP, Commodity Market, NBFC Schemes, Live Stock, Real Estate, Chit Funds, Shares, and Credit Society. It means that an alternative hypothesis is accepted that there is significant difference into investment pattern on the basis of of Educational Qualification of respondents.

\section{Hypothesis Testing for Occupation and Investment pattern}

Hypothesis related to Occupation is tested with the help of ANOVA

Following table shows ANOVA table for existing investment in investment instruments on the basis of Occupation. 


\begin{tabular}{|c|c|c|c|c|c|c|c|}
\hline \multicolumn{8}{|c|}{ Table 7: Occupation wise Investment in Instruments ANOVA } \\
\hline S.N & \multicolumn{2}{|c|}{ Particulars } & \multirow{2}{*}{$\begin{array}{l}\begin{array}{l}\text { Sum of } \\
\text { Squares }\end{array} \\
519.0\end{array}$} & \multirow{2}{*}{$\begin{array}{r}\text { df } \\
11\end{array}$} & \multirow{2}{*}{$\begin{array}{r}\begin{array}{c}\text { Mean } \\
\text { Square }\end{array} \\
47.2\end{array}$} & \multirow{2}{*}{$\begin{array}{l}\mathrm{F} \\
3.3\end{array}$} & \multirow{2}{*}{$\begin{array}{l}\text { Sig. } \\
.000\end{array}$} \\
\hline \multirow{3}{*}{1} & \multirow{3}{*}{ NSC } & Between Groups & & & & & \\
\hline & & Within Groups & 9902.2 & 698 & 14.2 & & \\
\hline & & Total & 10421.2 & 709 & & & \\
\hline \multirow{3}{*}{2} & \multirow{3}{*}{ PPF } & Between Groups & 9778.2 & 11 & 888.9 & 8.4 & .000 \\
\hline & & Within Groups & 74063.4 & 698 & 106.1 & & \\
\hline & & Total & 83841.5 & 709 & & & \\
\hline \multirow{3}{*}{3} & \multirow{3}{*}{$\begin{array}{l}\text { Bank Fixed } \\
\text { Deposits }\end{array}$} & Between Groups & 89242.8 & 11 & 8113.0 & 12.4 & .000 \\
\hline & & Within Groups & 456256.3 & 698 & 653.7 & & \\
\hline & & Total & 545499.2 & 709 & & & \\
\hline \multirow{3}{*}{4} & \multirow{3}{*}{ PO Schemes } & Between Groups & 9765.6 & 11 & 887.8 & 8.6 & .000 \\
\hline & & Within Groups & 72259.9 & 698 & 103.5 & & \\
\hline & & Total & 82025.5 & 709 & & & \\
\hline \multirow{3}{*}{5} & \multirow{3}{*}{$\begin{array}{l}\text { Govt. } \\
\text { Securities }\end{array}$} & Between Groups & 488.4 & 11 & 44.4 & 1.9 & .033 \\
\hline & & Within Groups & 16066.8 & 698 & 23.0 & & \\
\hline & & Total & 16555.2 & 709 & & & \\
\hline \multirow{3}{*}{6} & \multirow{3}{*}{ Insurance } & Between Groups & 8581.4 & 11 & 780.1 & 2.6 & .003 \\
\hline & & Within Groups & 206395.4 & 698 & 295.7 & & \\
\hline & & Total & 214976.8 & 709 & & & \\
\hline \multirow{3}{*}{7} & \multirow{3}{*}{ Mutual Funds } & Between Groups & 2978.5 & 11 & 270.8 & 6.6 & .000 \\
\hline & & Within Groups & 28828.0 & 698 & 41.3 & & \\
\hline & & Total & 31806.5 & 709 & & & \\
\hline \multirow{2}{*}{8} & \multirow{2}{*}{ ELSS } & Between Groups & 75.5 & 11 & 6.9 & 1.8 & .056 \\
\hline & & Within Groups & 2712.3 & 698 & 3.9 & & \\
\hline
\end{tabular}




\begin{tabular}{|c|c|c|c|c|c|c|c|}
\hline & & Total & 2787.9 & 709 & & & \\
\hline & \multirow{3}{*}{ Debentures } & Between Groups & 51.5 & 11 & 4.7 & .6 & .866 \\
\hline 9 & & Within Groups & 5895.1 & 698 & 8.4 & & \\
\hline & & Total & 5946.6 & 709 & & & \\
\hline \multirow{3}{*}{10} & \multirow{3}{*}{ Bonds } & Between Groups & $\begin{array}{l}950.1 \\
\end{array}$ & 11 & 86.4 & 6.5 & .000 \\
\hline & & Within Groups & 9241.4 & 698 & 13.2 & & \\
\hline & & Total & 10191.5 & 709 & & & \\
\hline \multirow{3}{*}{11} & \multirow{3}{*}{ Gold/Silver } & Between Groups & 9760.6 & 11 & 887.3 & 7.5 & .000 \\
\hline & & Within Groups & 82466.2 & 698 & 118.1 & & \\
\hline & & Total & 92226.8 & 709 & & & \\
\hline \multirow{3}{*}{12} & \multirow{3}{*}{$\begin{array}{l}\text { Company } \\
\text { Deposits }\end{array}$} & Between Groups & 157.0 & 11 & 14.3 & 2.2 & 012 \\
\hline & & Within Groups & 4480.9 & 698 & 6.4 & & \\
\hline & & Total & 4637.9 & 709 & & & \\
\hline \multirow{3}{*}{13} & \multirow{3}{*}{ SIP } & Between Groups & 3002.0 & 11 & 272.9 & 5.0 & .000 \\
\hline & & Within Groups & 38326.3 & 698 & 54.9 & & \\
\hline & & Total & 41328.3 & 709 & & & \\
\hline \multirow{3}{*}{14} & \multirow{3}{*}{ ULIP } & Between Groups & 284.3 & 11 & 25.8 & 3.1 & .000 \\
\hline & & Within Groups & 5863.6 & 698 & 8.4 & & \\
\hline & & Total & 6147.9 & 709 & & & \\
\hline \multirow{3}{*}{15} & \multirow{3}{*}{$\begin{array}{l}\text { Commodity } \\
\text { Market }\end{array}$} & Between Groups & 138.8 & 11 & 12.6 & 1.6 & .082 \\
\hline & & Within Groups & 5355.2 & 698 & 7.7 & & \\
\hline & & Total & 5494.0 & 709 & & & \\
\hline \multirow{3}{*}{16} & \multirow{3}{*}{ NBFC Scheme: } & Between Groups & 3.5 & 11 & .3 & 1.1 & .343 \\
\hline & & Within Groups & 196.0 & 698 & .3 & & \\
\hline & & Total & 199.4 & 709 & & & \\
\hline 17 & Live Stock & Between Groups & 6974.0 & 11 & 634.0 & 9.7 & .000 \\
\hline
\end{tabular}




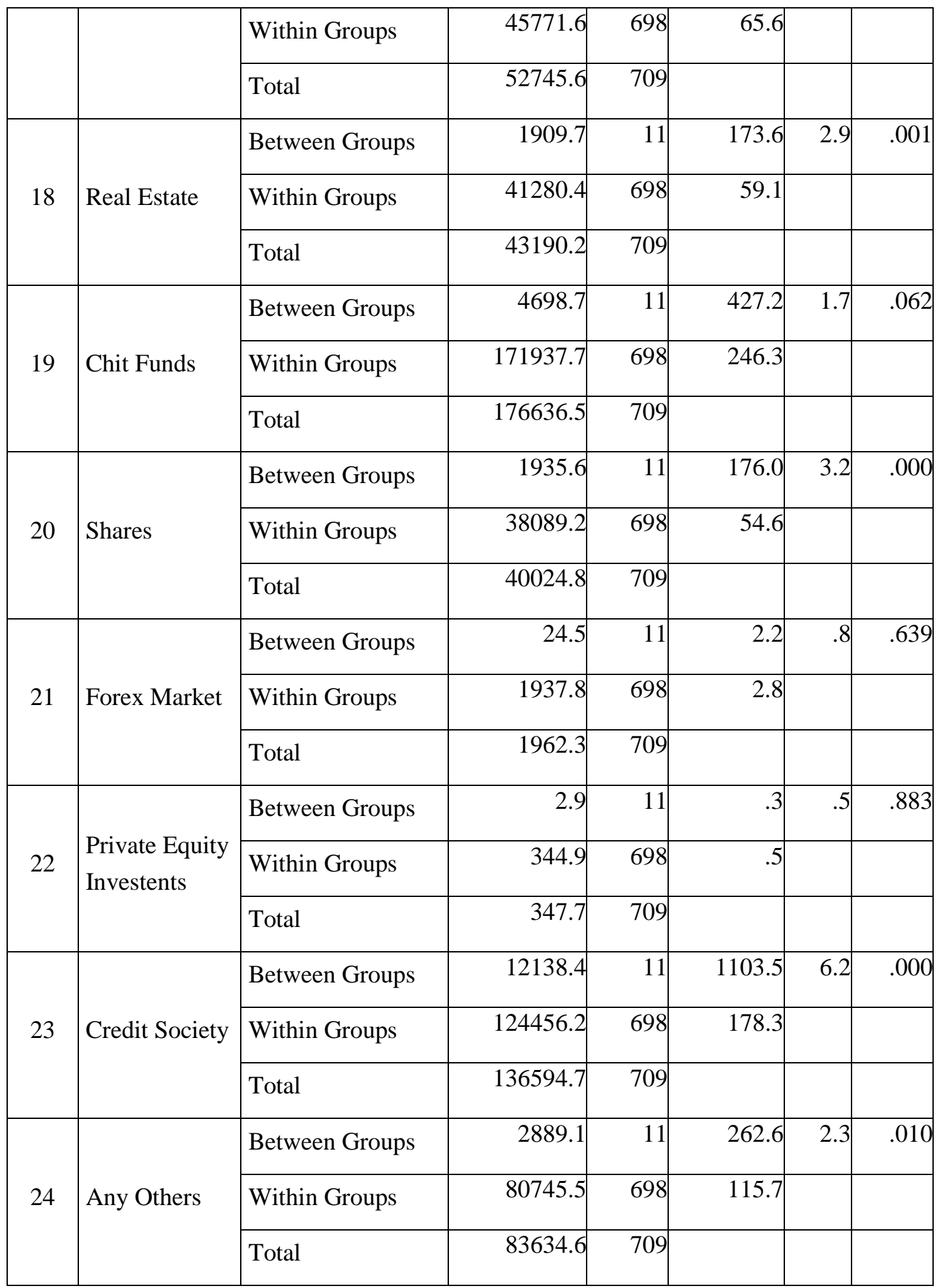

Source: (Compiled by Researcher)

Table 7 shows that ANOVA model is significant with investment instruments namely NSC, PPF, Bank Deposits, PO Schemes, Government Securities, Insurance, Mutual Funds, Bonds, Gold/Silver, SIP, ULIP, Live Stock, Real Estate, Shares, and Credit Society. These instruments are significant at $95 \%$ confidence level. 
To verify the results of ANOVA, Kruskal Wallis Test is also performed.

Table 8: Kruskal Wallis Test between Investment Instruments

\begin{tabular}{|c|c|c|c|c|}
\hline S.N & Investment Avenues & Chi-Square & df & Asymp. Sig. \\
\hline 1 & NSC & 40.98 & 11 & .00 \\
\hline 2 & PPF & 97.81 & 11 & .00 \\
\hline 3 & Bank Fixed Deposits & 110.66 & 11 & .00 \\
\hline 4 & PO Schemes & 91.73 & 11 & .00 \\
\hline 5 & Government Securities & 27.05 & 11 & .00 \\
\hline 6 & Insurance & 67.99 & 11 & .00 \\
\hline 7 & Mutual Funds & 86.06 & 11 & .00 \\
\hline 8 & ELSS & 17.91 & 11 & .08 \\
\hline 9 & Debentures & 12.03 & 11 & .36 \\
\hline 10 & Bonds & 70.64 & 11 & .00 \\
\hline 11 & Gold/ Silver & 89.62 & 11 & .00 \\
\hline 12 & Company Deposits & 33.97 & 11 & .00 \\
\hline 13 & SIP & 66.01 & 11 & .00 \\
\hline 14 & ULIP & 34.39 & 11 & .00 \\
\hline 15 & Commodity Market & 20.40 & 11 & .04 \\
\hline 16 & NBFC Schemes & 12.29 & 11 & .34 \\
\hline 17 & Live Stock & 102.25 & 11 & .00 \\
\hline 18 & Real Estate & 48.84 & 11 & .00 \\
\hline 19 & Chit Funds & 18.12 & 11 & .08 \\
\hline 20 & Shares & 50.14 & 11 & .00 \\
\hline 21 & Forex Market & 8.46 & 11 & .67 \\
\hline 22 & Private Equity & 6.09 & 11 & .87 \\
\hline 23 & Credit Society & 56.01 & 11 & .00 \\
\hline 24 & Any Other & 93.43 & 11 & .00 \\
\hline
\end{tabular}

$(\mathrm{n}=710)$

Source: (Compiled by Researcher) 
Table 8 shows that Kruskal wallis is significant with investment instruments namely NSC, PPF, Bank Deposits, PO Schemes, Government Securities, Insurance, Mutual Funds, ELSS, Bonds, Gold/Silver, Company Deposits, SIP, ULIP, Commodity Market, Live Stock, Real Estate, Chit Funds, Shares, and Credit Society. These instruments are significant at 95\% confidence level. It means that the results of ANOVA and Kruskal Wallis test shows that null hypotheses is rejected with investment instruments viz. NSC, PPF, Bank Deposits, PO Schemes, Government Securities, Insurance, Mutual Funds, Bonds, Gold/Silver, SIP, ULIP, Live Stock, Real Estate, Shares, and Credit Society. It means that an alternative hypothesis is accepted that there is significant difference into investment pattern on the basis of Occupation of respondents.

\section{Hypothesis Testing for Income group and Investment pattern}

Hypothesis related to Income group is tested with the help of ANOVA

Following table shows ANOVA testing of the investment in instruments as per Income group of sample investors. 


\begin{tabular}{|c|c|c|c|c|c|c|c|}
\hline \multicolumn{8}{|c|}{ Table 9: Income Group wise Investment in Instruments ANOVA } \\
\hline S.N & \multicolumn{2}{|c|}{ Particulars } & \multirow{2}{*}{$\begin{array}{c}\text { Sum of } \\
\text { Squares }\end{array}$} & \multirow{2}{*}{$\begin{array}{l}\text { df } \\
4\end{array}$} & \multirow{2}{*}{$\begin{array}{c}\text { Mean } \\
\text { Square }\end{array}$} & \multirow{2}{*}{$\begin{array}{c}\mathrm{F} \\
18.9\end{array}$} & \multirow{2}{*}{$\begin{array}{l}\text { Sig. } \\
.000\end{array}$} \\
\hline \multirow{3}{*}{1} & \multirow{3}{*}{ NSC } & Between Groups & & & & & \\
\hline & & Within Groups & 9413.3 & 705 & 13.4 & & \\
\hline & & Total & 10421.2 & 709 & & & \\
\hline \multirow{3}{*}{2} & \multirow{3}{*}{ PPF } & Between Groups & 5608.3 & 4 & 1402.1 & 12.6 & .000 \\
\hline & & Within Groups & 78233.2 & 705 & 111.0 & & \\
\hline & & Total & 83841.5 & 709 & & & \\
\hline \multirow{3}{*}{3} & \multirow{3}{*}{$\begin{array}{c}\text { Bank Fixed } \\
\text { Deposits }\end{array}$} & Between Groups & 85515.8 & 4 & 21378.9 & 32.8 & .000 \\
\hline & & Within Groups & 459983.4 & 705 & 652.5 & & \\
\hline & & Total & 545499.2 & 709 & & & \\
\hline \multirow{3}{*}{4} & \multirow{3}{*}{ PO Schemes } & Between Groups & 2810.3 & 4 & 702.6 & 6.3 & .000 \\
\hline & & Within Groups & 79215.2 & 705 & 112.4 & & \\
\hline & & Total & 82025.5 & 709 & & & \\
\hline \multirow{3}{*}{5} & \multirow{3}{*}{ Govt. Securities } & Between Groups & 352.5 & 4 & 88.1 & 3.8 & .004 \\
\hline & & Within Groups & 16202.7 & 705 & 23.0 & & \\
\hline & & Total & 16555.2 & 709 & & & \\
\hline \multirow{3}{*}{6} & \multirow{3}{*}{ Insurance } & Between Groups & 5110.9 & 4 & 1277.7 & 4.3 & .002 \\
\hline & & Within Groups & 209865.9 & 705 & 297.7 & & \\
\hline & & Total & 214976.8 & 709 & & & \\
\hline \multirow{3}{*}{7} & \multirow{3}{*}{ Mutual Funds } & Between Groups & 5556.1 & 4 & 1389.0 & 37.3 & .000 \\
\hline & & Within Groups & 26250.4 & 705 & 37.2 & & \\
\hline & & Total & 31806.5 & 709 & & & \\
\hline \multirow{2}{*}{8} & \multirow{2}{*}{ ELSS } & Between Groups & 53.1 & 4 & 13.3 & 3.4 & .009 \\
\hline & & Within Groups & 2734.8 & 705 & 3.9 & & \\
\hline
\end{tabular}




\begin{tabular}{|c|c|c|c|c|c|c|c|}
\hline & & Total & 2787.9 & 709 & & & \\
\hline \multirow{3}{*}{9} & \multirow{3}{*}{ Debentures } & Between Groups & 56.5 & 4 & 14.1 & 1.7 & .150 \\
\hline & & Within Groups & 5890.1 & 705 & 8.4 & & \\
\hline & & Total & 5946.6 & 709 & & & \\
\hline \multirow{3}{*}{10} & \multirow{3}{*}{ Bonds } & Between Groups & 2249.2 & 4 & 562.3 & 49.9 & .000 \\
\hline & & Within Groups & 7942.3 & 705 & 11.3 & & \\
\hline & & Total & 10191.5 & 709 & & & \\
\hline \multirow{3}{*}{11} & \multirow{3}{*}{ Gold/Silver } & Between Groups & 3590.3 & 4 & 897.6 & 7.1 & .000 \\
\hline & & Within Groups & 88636.5 & 705 & 125.7 & & \\
\hline & & Total & 92226.8 & 709 & & & \\
\hline \multirow{3}{*}{12} & \multirow{3}{*}{$\begin{array}{l}\text { Company } \\
\text { Deposits }\end{array}$} & Between Groups & 37.4 & 4 & 9.4 & 1.4 & .221 \\
\hline & & Within Groups & 4600.5 & 705 & 6.5 & & \\
\hline & & Total & 4637.9 & 709 & & & \\
\hline \multirow{3}{*}{13} & \multirow{3}{*}{ SIP } & Between Groups & 2563.0 & 4 & 640.8 & 11.7 & .000 \\
\hline & & Within Groups & 38765.3 & 705 & 55.0 & & \\
\hline & & Total & 41328.3 & 709 & & & \\
\hline \multirow{3}{*}{14} & \multirow{3}{*}{ ULIP } & Between Groups & 538.8 & 4 & 134.7 & 16.9 & .000 \\
\hline & & Within Groups & 5609.1 & 705 & 8.0 & & \\
\hline & & Total & 6147.9 & 709 & & & \\
\hline \multirow{3}{*}{15} & \multirow{3}{*}{$\begin{array}{c}\text { Commodity } \\
\text { Market }\end{array}$} & Between Groups & 340.0 & 4 & 85.0 & 11.6 & .000 \\
\hline & & Within Groups & 5154.0 & 705 & 7.3 & & \\
\hline & & Total & 5494.0 & 709 & & & \\
\hline \multirow{3}{*}{16} & \multirow{3}{*}{ NBFC Schemes } & Between Groups & .4 & 4 & .1 & .4 & .827 \\
\hline & & Within Groups & 199.0 & 705 & .3 & & \\
\hline & & Total & 199.4 & 709 & & & \\
\hline 17 & Live Stock & Between Groups & 1714.5 & 4 & 428.6 & 5.9 & .000 \\
\hline
\end{tabular}




\begin{tabular}{|c|c|c|c|c|c|c|c|}
\hline & & Within Groups & 51031.1 & 705 & 72.4 & & \\
\hline & & Total & 52745.6 & 709 & & & \\
\hline & & Between Groups & 671.3 & 4 & 167.8 & 2.8 & .026 \\
\hline 18 & Real Estate & Within Groups & 42518.9 & 705 & 60.3 & & \\
\hline & & Total & 43190.2 & 709 & & & \\
\hline & & Between Groups & 1686.5 & 4 & 421.6 & 1.7 & .148 \\
\hline 19 & Chit Funds & Within Groups & 174949.9 & 705 & 248.2 & & \\
\hline & & Total & 176636.5 & 709 & & & \\
\hline & & Between Groups & 5125.0 & 4 & 1281.2 & 25.9 & .000 \\
\hline 20 & Shares & Within Groups & 34899.8 & 705 & 49.5 & & \\
\hline & & Total & 40024.8 & 709 & & & \\
\hline & & Between Groups & 65.7 & 4 & 16.4 & 6.1 & .000 \\
\hline 21 & Forex Market & Within Groups & 1896.6 & 705 & 2.7 & & \\
\hline & & Total & 1962.3 & 709 & & & \\
\hline & & Between Groups & 1.1 & 4 & .3 & .5 & .704 \\
\hline 22 & Private Equity & Within Groups & 346.7 & 705 & .5 & & \\
\hline & & Total & 347.7 & 709 & & & \\
\hline & & Between Groups & 6771.1 & 4 & 1692.8 & 9.2 & .000 \\
\hline 23 & Credit Society & Within Groups & 129823.6 & 705 & 184.1 & & \\
\hline & & Total & 136594.7 & 709 & & & \\
\hline & & Between Groups & 697.8 & 4 & 174.5 & 1.5 & .206 \\
\hline 24 & Any Others & Within Groups & 82936.8 & 705 & 117.6 & & \\
\hline & & Total & 83634.6 & 709 & & & \\
\hline
\end{tabular}

Source: (Compiled by Researcher)

Table: 9 shows that ANOVA model is significant with investment instruments namely NSC, PPF, Bank Deposits, PO Schemes, Government Securities, Insurance, Mutual Funds, ELSS, Bonds, Gold/Silver, SIP, ULIP, Commodity Market, Live Stock, Real Estate, Shares, Forex Market and Credit Society. These instruments are significant at $95 \%$ confidence level. 
To verify the results of ANOVA, Kruskal Wallis Test is also performed.

Table 10: Kruskal Wallis Test between Investment Instruments

\begin{tabular}{|c|c|c|c|c|}
\hline S.N & Investment Avenues & Chi-Square & Df & Asymp. Sig. \\
\hline 1 & NSC & 87.2 & 4 & .00 \\
\hline 2 & PPF & 81.0 & 4 & .00 \\
\hline 3 & Bank Fixed Deposits & 89.5 & 4 & .00 \\
\hline 4 & PO Schemes & 15.5 & 4 & .00 \\
\hline 5 & Government Securities & 18.7 & 4 & .00 \\
\hline 6 & Insurance & 70.9 & 4 & .00 \\
\hline 7 & Mutual Funds & 169.5 & 4 & .00 \\
\hline 8 & ELSS & 16.9 & 4 & .00 \\
\hline 9 & Debentures & 10.3 & 4 & .04 \\
\hline 10 & Bonds & 166.3 & 4 & .00 \\
\hline 11 & Gold/ Silver & 35.9 & 4 & .00 \\
\hline 12 & Company Deposits & 3.9 & 4 & .42 \\
\hline 13 & SIP & 114.4 & 4 & .00 \\
\hline 14 & ULIP & 65.8 & 4 & .00 \\
\hline 15 & Commodity Market & 39.1 & 4 & .00 \\
\hline 16 & NBFC Schemes & 1.5 & 4 & .83 \\
\hline 17 & Live Stock & 24.6 & 4 & .00 \\
\hline 18 & Real Estate & 19.2 & 4 & .00 \\
\hline 19 & Chit Funds & 4.4 & 4 & .35 \\
\hline 20 & Shares & 132.8 & 4 & .00 \\
\hline 21 & Forex Market & 26.6 & 4 & .00 \\
\hline 22 & Private Equity & 2.0 & 4 & .74 \\
\hline 23 & Credit Society & 19.3 & 4 & .00 \\
\hline 24 & Any Other & 7.9 & 4 & .10 \\
\hline
\end{tabular}

Source: (Compiled by Researcher) 
Table 10 shows that Kruskal wallis model is significant with investment instruments namely NSC, PPF, Bank Deposits, PO Schemes, Government Securities, Insurance, Mutual Funds, ELSS, Debentures, Bonds, Gold/Silver, SIP, ULIP, Commodity Market, Live Stock, Real Estate, Chit Funds, Shares, Forex Market and Credit Society. These instruments are significant at $95 \%$ confidence level.

It means that the results of ANOVA and Kruskal Wallis test shows that null hypotheses is rejected with investment instruments viz. NSC, PPF, Bank Deposits, PO Schemes, Government Securities, Insurance, Mutual Funds, ELSS, Bonds, Gold/Silver, SIP, ULIP, Commodity Market, Live Stock, Real Estate, Shares, Forex Market and Credit Society. It means that an alternative hypothesis is accepted that there is significant difference into investment pattern on the basis of Income group of respondents.

\section{MAJOR FINDINGS}

It is found that by using parametric test such as Independent sample ' $t$ " test and ANOVA and nonparametric test such as Mann-Whitney Test and Kruskal Wallis Test, there is significant difference in investment pattern on the magnitude of demographic factors such as Age, Income and Educational Qualification.

\section{CONCLUSION}

The study on people's choice in Investment Choices has been undertaken with the objective, to analyze the investment pattern of investors on the magnitude of demographic traits all the age groups give more preference to invest in Insurance, NSC, PPF and Bank Deposit. Income level of a respondent is also an important factor which affects portfolio of the respondent. Middle age group, Lower income level groups respondents are preferred to invest in Insurance, NSC, PPF and bank deposit rather than any other investment avenues. The purpose of this study was to determine whether the variables such as demographic characteristics (age, gender) and investment patterns could be used individually or in combination to both differentiate among levels of men and women investment decisions and risk tolerance and develop some guidelines to the investment managers to design their investment schemes by considering these views of individuals.

\section{REFERENCES}

[1] Croson, R. Gneezy, U. (2009). Gender differences in preferences. Journal of Economic Literature, 47(2), 1-27.

[2] Das B., M. S. (2008). Mutual Fund vs. Life Insurance: Behavioural Analysis of Retail Investors. International Journal of Business and Management, 3 (10).

[3] Eckel, C. C. (2001). Chivalry and solidarity in Ultimatum Games. Economic Inquiry, 39 (2), 171-188.

[4] Giridhari Mohanta, Sathya Swaroop Debasish (2011) A Study on Investment Preferences among Urban Investors in Orissa. Prerna Journal of Management Thought and Practice, 3(1), $1-9$

[5] Gnana Design.C, Kalaiselvi. S, Anusya.L (2006), Women Investors Perception towards Investment - An Empirical Study, Indian Journal of Marketing, 36(4), 14-37 
[6] Karthikeyan B. (2001). Small Investors' Perception on Post Office Small Savings Schemes unpublished thesis, MadrasUniversity, Tamilnadu, India.

[7] Kruse, J. B. and M. A. Thompson (2003) Valuing Low Probability Risk: survey and experimental evidence, Journal of Economic Behavior and Organization, 50(4)

[8] Manish Mittal and R K Vyas, (2007). Demographics and Investment Choice among Indian Investors". Review of Business and Technology Research, 3 (1), 51-65.

[9] Mittal Manish, R. (2008). Personality Type and Investment Choice: An Empirical Study. TheIUP Journal of Behavioural Finance, Vol. V, No. 3, 6-22.

[10] Meenu Verma (2008), Wealth Management and Behavioral Finance: The Effect of Demographics and Personality on Investment Choice among Indian Investors", The IUP Journal of Behavioral Finance, $P$ - 20

[11] Rajarajan (2002), "Determinants of Portfolio Choice of Individual Investors", Indian Economic Journal, 81-84.

[12] Rajarajan, V (2000), "Investors Life Cycle and Investment Characteristics", Finance India, 14(2). 465-478.

[13] Rajarajan,V (2003), "Investors Demographics and Risk Bearing Capacity", Finance India, 17(2), 565-576.

[14] Rajarajan.V (1997), “Investment Size Based Segmentation of Individual Investor", Management Researcher, 3(4), 21-28.

[15] Schubert, R., Brown, M., Gysler, M. and Brachinger, H. W. (1999). Financial decision making: are women really more risk-averse? American Economic Review, 89(2), 381- 385.

[16] Srinivasan Sakthi K., L. D. (2006). Post Office Savings Schemes - An Impetus for Rural Investment. Indinan Journal of Marketing, 36(1), 22-37.

[17] Suman and D. P. Warne (2012) Investment Behavior of Individual Investor in Stock Market., IJRFM , 2(2) 